\title{
Associations between subjective social status and physical and mental health functioning among patients with hypertension
}

\author{
Crystal W Cené', Jacqueline R Halladay', \\ Ziya Gizlice', Kyle Roedersheimer', \\ Alan Hinderliter', Doyle M Cummings², \\ Katrina E Donahue', Andrew J Perrin' and \\ Darren A DeWalt'
}

\begin{abstract}
We examine the cross-sectional association between subjective social status and self-rated physical and mental health functioning in 518 Black and White patients enrolled in a community-based hypertension control research study. We found that (I) subjective social status, measured using both a proximal and distal referent group, was positively associated with physical and mental health functioning scores independent of educational level, household income, or both; (2) the effect of subjective social status on physical and mental health functioning differed significantly by race when using the distal, not the proximal, referent group. When the associations differed, they were stronger for Whites than Blacks.
\end{abstract}

\section{Keywords}

adults, hypertension, quality of life, race, socio-economic status

\section{Background}

Socioeconomic status (SES) is a measure of social status, which profoundly impacts health by structuring an individual's access to both material (e.g. financial assets, healthcare) and social (e.g. influential social networks) resources required to achieve and maintain good health. Most studies use objective social status (OSS) measures, such as educational level, income, occupation, or wealth to assess SES. However, subjective social status (SSS) may be a more sensitive measure of SES than OSS indicators (Wolff et al., 2010). SSS captures how individuals perceive their relative position in the social hierarchy and may more accurately assess the cumulative effect of one's relative social position on health. In rating SSS,

\footnotetext{
IUniversity of North Carolina at Chapel Hill, USA 2East Carolina University, USA

Corresponding author:

Crystal W Cené, Cecil G. Sheps Center for Health Services Research, Department of Medicine, University of North Carolina at Chapel Hill, 5034 Old Clinic Building, CB\# 7I 10, Chapel Hill, NC 27599, USA.

Email: crystal_cene@med.unc.edu
} 
individuals consider more than just their relative standing on the various OSS components (Adler et al., 2008); they also consider past circumstances and experiences (e.g. educational quality), family history and resources (e.g. wealth of extended family), future prospects and opportunities, as well as psychological factors (e.g. selfesteem, respect from peers) that affect health trajectories (Franzini and Fernandez-Esquer, 2006; Hu et al., 2005; Operario et al., 2004; Singh-Manoux et al., 2005; Wright and Steptoe, 2005). In essence, SSS is more than simply how much one has; rather, it is also how much one believes he has relative to others (Kraus and Stephens, 2012).

Low SSS has been associated with a variety of health outcomes, including poorer self-rated health (Franzini and Fernandez-Esquer, 2006; $\mathrm{Hu}$ et al., 2005; Operario et al., 2004; SinghManoux et al., 2005; Wright and Steptoe, 2005), worse mental health (Franzini and FernandezEsquer, 2006; Singh-Manoux et al., 2005), worse subjective well-being (Anderson et al., 2012), harmful health behaviors such as smoking (Finkelstein et al., 2006) and medical conditions including diabetes, depression, hypertension, angina, and respiratory illnesses (Adler et al., 2008). While most research to date has been cross-sectional, several prospective studies have also demonstrated that SSS explains changes in health status (Cohen et al., 2008; Goodman et al., 2007; Singh-Manoux et al., 2005; Thompson et al., 2014). Many of the associations between SSS and health outcomes remain after adjusting for OSS measures, suggesting that SSS independently affects health (Cornman et al., 2003; Franzini and Fernandez-Esquer, 2006; Hu et al., 2005; Operario et al., 2004; Ostrove et al., 2000; Singh-Manoux et al., 2005; Wright and Steptoe, 2005) and is not just a proxy for OSS.

Most studies use a single distal referent group ("others in society") when making SSS comparisons; however, some research suggests that the referent group used for comparison (e.g. proximal- "others in your community" vs distal"others in the United States") differentially affects SSS ratings among Whites, Blacks, and Hispanics (Wolff et al., 2010). However, few studies have assessed SSS using both proximal and distal referent groups and examined their relationship to health-related outcomes (Subramanyam et al., 2012). The aims of our study are to (1) assess Black-White differences in SSS using both proximal (community) and distal (US) referent groups, (2) examine the correlation between OSS indicators (education and annual income) and SSS measures in Blacks and Whites, (3) examine the association between SSS measures and self-rated physical and mental health functioning in race-stratified samples, and (4) assess whether observed associations persist after adjusting for education and income, among a rural community-dwelling sample of patients with hypertension. We hypothesize that (1) racial differences in SSS will depend on the referent group, such that Blacks will have higher SSS ratings than Whites using the community versus the US referent groups, (2) correlations between the OSS and SSS measures will be stronger among Whites than among Blacks, (3) SSS will be significantly associated with both physical and mental health functioning, and (4) associations between SSS, physical, and mental health functioning will be attenuated, but persist, after adjusting for education and income.

\section{Methods}

\section{Participants and sampling procedures}

We conducted a secondary analysis of baseline data from 535 study participants enrolled in an ongoing 5-year cardiovascular risk reduction study in a rural, economically distressed county in the Southeastern United States. A detailed description of the study has been previously published (Halladay et al., 2013). Study participants were eligible if they had a diagnosis of hypertension by a primary care provider or had three documented blood pressure measurements above $150 / 90 \mathrm{mmHg}$, had a systolic blood pressure of $\geqslant 150 \mathrm{mmHg}$ at their most recent clinic visit, and were 18 years or older. For this article, we limited our analytic sample to participants with self-reported African American/Black or White race and those with 


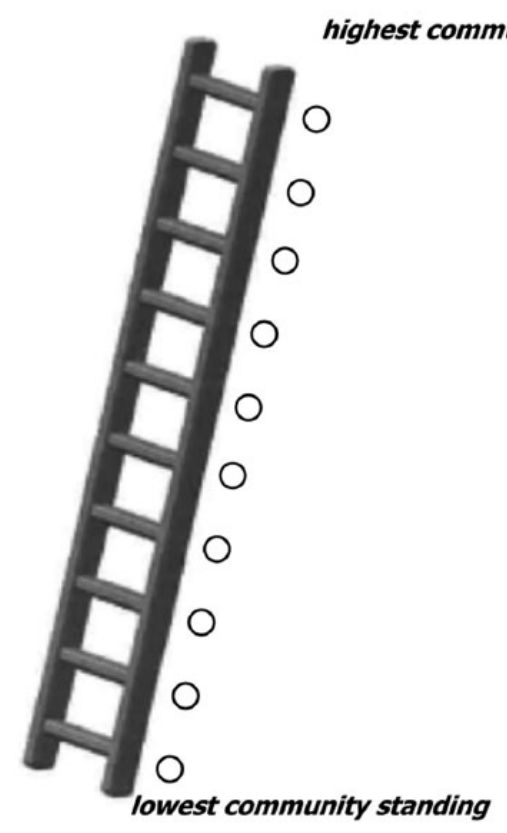

Figure I. MacArthur social ladder scales.

data on the SSS measures, yielding an analytic sample size of 518 . The study was approved by the Biomedical Institutional Review Board at The University of North Carolina at Chapel Hill.

\section{Measures}

SSS. We used the MacArthur scale - a selfanchoring scale using a pictorial format to present a 10-rung "social ladder" (MacArthur Research Network on Socioeconomic Status and Health, 2008) which allows individuals to consider the special social circumstances of their life to more accurately reflect their social status. Respondents are asked to place an " $\mathrm{X}$ " on the rung where they feel they stand. We used two versions of the ladder (Figure 1), one linked to traditional OSS indicators ("US ladder"; distal referent) and the second linked to standing in one's community ("community ladder"; proximal referent). The differences between the two ladders is of particular interest in poorer communities in which individuals may not be

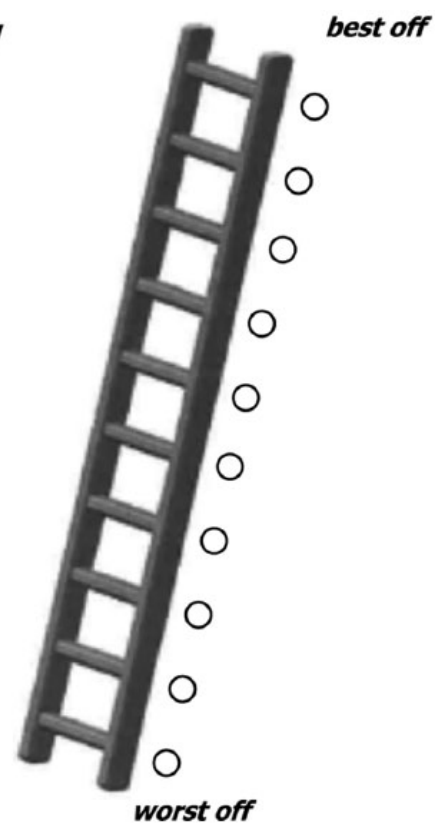

advantaged in terms of income, occupation, or education, but may have high standing within their local community and among their social networks.

OSS. Two objective measures of SES were assessed: highest educational level in years and annual household income (measured in 13 categories, ranging from $<$ US $\$ 5000$ to $\geqslant U S \$ 100,000$ and further collapsed into $\geqslant U S \$ 40,000$, $<\mathrm{US} \$ 40,000$, and missing as a category in regression analyses).

Physical and mental health functioning. These outcome variables were assessed using the short-form 12 (SF-12; standard 4-week recall format), a well validated measure of healthrelated quality of life (Ware et al., 1996) (See Supplementary Material online). This measure includes two subscores, a physical component summary (PCS) score and a mental component summary (MCS) score with standardized scores from 0 to 100 , based on weighted item response categories from each of the 12 questions (Ware 
et al., 1996). Low scores indicate poor functioning; 50 is the mean in the general US population. Cronbach's alpha (internal reliability) coefficients for the PCS and MCS were 0.68 and 0.60 , respectively, in this sample.

Race. Race was self-reported with categories consistent with the US Census. Nine participants self-identified as either White or Black and another race and were therefore categorized as either White or Black and not the other race they listed (e.g. American Indian) for analysis purposes. No participants listed their race as White and Black. Nine participants reported Hispanic/Latino ethnicity. Five of the nine listed their race as either White or Black and were analyzed according to their race group. The other four were excluded from the race derived variable because they did not list a race.

Covariates. Other self-reported covariates of interest included marital status, dichotomized as currently married/partnered versus not, and employment status, dichotomized as currently employed (including working full- or part-time) versus not currently employed.

\section{Statistical analysis}

We used descriptive statistics such as means, percentages, and standard errors to summarize and compare study characteristics between two race groups using Chi-square tests for categorical variables and $t$-tests for continuous variables. We fit a set of sequential linear regression models to examine the independent associations between each SSS ladder (community and US standing) and the health outcomes (PCS and MCS), adjusting for education level and household income. The initial model included: age, marital status, and employment status as control variables, since these variables are theoretically relevant and have been associated with both SSS and OSS in prior research (Kaufman et al., 1998), and an SSS measure. Then, we fit two separate models by adding education level and household income as covariates to the initial model. The final model included age, marital status, employment status, educational level, income, and the SSS measure. We present findings for the overall sample, as well as stratified by race using the same four models described above. To examine whether the association of each SSS measure and our outcomes (PCS and MCS scores) differed by race, we included race and a race by SSS measure interaction term in the model. In supplemental analyses, we also tested for interactions between race and each OSS indicator (educational level and income) for each outcome measure to examine whether any observed associations might be explained by racial differences in OSS measures. As an exploratory study, we did not adjust $p$ values for testing multiple hypotheses. We consider a $p$ value of $<0.05$ as statistically significant.

\section{Results}

\section{Sample description}

Table 1 presents the sample characteristics. Overall, 59 percent of the sample was Black and 32 percent were males. The mean age was 58 years, and Whites were on average about 2 years older than Blacks. On average, Whites reported a higher educational level than Blacks and significantly more Whites reported an annual household income $\geqslant U S \$ 40,000$. Mean SSS scores were higher for Blacks than Whites using both community ( 6.8 vs $6.1 ; p=0.01)$ and US (5.6 vs 5.2; $p=0.03$ ) referent groups, respectively. The mean PCS (40.8 vs 39.0) and MCS (49.4 vs 49.0) scores were not significantly different for Blacks and Whites, respectively.

\section{SSS and OSS}

Correlations between OSS and SSS by race are presented in Table 2. Among Blacks, only the correlation between community SSS and income was significant. Among Whites, both community and US SSS were significantly correlated with education and income. The racial differences were statistically significant for only the correlations with US SSS, not community SSS. 
Table I. Sociodemographic and clinical characteristics of the sample overall and by race.

\begin{tabular}{|c|c|c|c|c|}
\hline & $\begin{array}{l}\text { Overall sample } \\
(N=5 \mid 8)\end{array}$ & $\begin{array}{l}\text { Black } \\
(n=306)\end{array}$ & $\begin{array}{l}\text { White } \\
(n=2 \mid 2)\end{array}$ & $p$ value \\
\hline Age (years), mean (SE) & $57.6(0.55)$ & $56.9(0.73)$ & $59.5(0.83)$ & 0.02 \\
\hline Gender, male (\%) & $166(32 \%)$ & $88(29 \%)$ & $78(37 \%)$ & 0.05 \\
\hline \multicolumn{5}{|l|}{ Marital status (\%) } \\
\hline Currently married/partnered & 237 (46\%) & $109(36 \%)$ & $128(60 \%)$ & $<0.0001$ \\
\hline Highest educational level, mean (SE) & $12.0(0.11)$ & $11.7(0.14)$ & $12.6(0.17)$ & $<0.0001$ \\
\hline \multicolumn{5}{|l|}{ Household income (\%) } \\
\hline$<$ US $\$ 40,000$ & $345(79 \%)$ & $224(88 \%)$ & $121(66 \%)$ & $<0.000$ I \\
\hline$\geqslant U S \$ 40,000$ & $94(21 \%)$ & $31(12 \%)$ & $63(34 \%)$ & \\
\hline DK/refused/missing & $80(15 \%)$ & $51(16)$ & $28(13)$ & \\
\hline \multicolumn{5}{|l|}{ Employed (\%) } \\
\hline Employed full or part time & $194(38 \%)$ & $120(39 \%)$ & $74(35 \%)$ & 0.32 \\
\hline PCS, mean (SE) $(N=510)$ & $40.0(0.5 \mathrm{I})$ & $40.7(0.63)$ & $39.0(0.84)$ & 0.14 \\
\hline MCS, mean (SE) $(N=5 I 0)$ & $49.2(0.48)$ & $49.3(0.60)$ & $48.9(0.79)$ & 0.91 \\
\hline Community SSS, mean (SE) & $6.5(0.10)$ & $6.8(0.13)$ & $6.2(0.16)$ & 0.01 \\
\hline US SSS, mean (SE) & $5.4(0.10)$ & $5.6(0.13)$ & $5.2(0.14)$ & 0.03 \\
\hline
\end{tabular}

SE: standard error; DK: don't know; SSS: subjective social status.

Table 2. Spearman correlations ( $p$ values) among OSS and SSS measures.

\begin{tabular}{|c|c|c|c|c|c|c|}
\hline & \multicolumn{2}{|l|}{ Blacks } & \multicolumn{2}{|l|}{ Whites } & \multicolumn{2}{|c|}{$\begin{array}{l}\text { Black-White differences (SE } \\
\text { of difference) in correlations }\end{array}$} \\
\hline & $\begin{array}{l}\text { Community } \\
\text { SSS }\end{array}$ & US SSS & $\begin{array}{l}\text { Community } \\
\text { SSS }\end{array}$ & US SSS & $\begin{array}{l}\text { Community } \\
\text { SSS }\end{array}$ & US SSS \\
\hline $\begin{array}{l}\text { Highest } \\
\text { education level } \\
\text { completed }^{a}\end{array}$ & $0.05(p=0.39)$ & $-0.02(p=0.76)$ & $0.15(p=0.03)$ & $0.23(p<0.001)$ & $\begin{array}{l}0.10(0.09) \\
(p=0.27)\end{array}$ & $0.25(0.09)^{*}$ \\
\hline $\begin{array}{l}\text { Annual } \\
\text { household } \\
\text { income }^{b}\end{array}$ & $0.14(p=0.02)$ & $0.11(p=0.08)$ & $0.27(p<0.001)$ & $0.43(p<0.0001)$ & $\begin{array}{l}0.13(0.10) \\
(p=0.18)\end{array}$ & $0.35(0.10)^{* * * *}$ \\
\hline
\end{tabular}

OSS: objective social status; SSS: subjective social status; SE: standard error.

aTreated as an ordinal variable.

bTreated as an ordinal categorical variable ranging from I $(<5000)$ to $13(100,000$ or more).

$* * *$ Between-group $p$ value $<0.001$; **between-group $p$ value $<0.01$; *between-group $p$ value $<0.05$.

\section{SSS and physical and mental health functioning using community referent group}

Overall, we found a significant association between community SSS and PCS score after adjusting for age, marital status, and employment status (Table 3, Model 1), such that physical health functioning score increased by 0.87 (SE $0.21 ; p<0.0001)$ for every 1 "rung" increase in community SSS. Community SSS remained significantly associated with higher PCS scores in the full model (Table 3, Model 3) $(\beta=0.81$; SE $0.21 ; p=0.0001)$. Although the effect estimates between community SSS and physical health functioning were greater in Whites than Blacks, the differences were not statistically different. Similarly, as community SSS increased, MCS scores increased by 0.71 (SE $0.20 ; p<0.001$; Model 1) after adjusting for age, marital status, 
Table 3. Linear associations between community standing and physical component summary (PCS) and mental component summary (MCS) scores, overall and race-stratified.

\begin{tabular}{|c|c|c|c|}
\hline & Overall & Blacks & Whites \\
\hline \multicolumn{4}{|l|}{ PCS score } \\
\hline \multicolumn{4}{|l|}{ Model I } \\
\hline Age & $0.02(0.04)$ & $-0.03(0.05)$ & $0.01(0.07)$ \\
\hline Not currently married & I.II (0.95) & $1.14(1.25)$ & $0.70(1.63)$ \\
\hline Not currently employed & $-7.26(1.02)$ & $-7.16(1.26)$ & $-6.96(1.76)$ \\
\hline Mean community SSS & $0.87(0.21)^{* * *}$ & $0.63(0.27)^{*}$ & $1.20(0.35)^{* *}$ \\
\hline \multicolumn{4}{|l|}{ Model 2a } \\
\hline Age & $-0.02(0.04)$ & $-0.02(0.05)$ & $0.01(0.07)$ \\
\hline Not currently married & $1.38(0.96)$ & $1.26(1.25)$ & $0.98(1.64)$ \\
\hline Not currently employed & $-7.09(1.02)$ & $-6.98(1.27)$ & $-6.73(1.76)$ \\
\hline Mean highest education (years) & $0.39(0.20)$ & $0.38(0.25)$ & $0.47(0.33)$ \\
\hline Mean community SSS & $0.83(0.21)^{* * *}$ & $0.59(.27)^{*}$ & $1.13(0.36)^{* *}$ \\
\hline \multicolumn{4}{|l|}{ Model $2 b$} \\
\hline Age & $-0.03(0.04)$ & $-0.03(0.05)$ & $0.01(0.07)$ \\
\hline Not currently married & $1.48(1.02)$ & $1.14(1.30)$ & $1.12(1.78)$ \\
\hline Not currently employed & $-7.13(1.06)$ & $-7.32(1.32)$ & $-6.45(1.83)$ \\
\hline \multicolumn{4}{|l|}{ Income ("missing"= referent) } \\
\hline$<$ US $\$ 40,000$ & $-1.33(1.38)$ & $-3.15(1.65)$ & $\mathrm{I} .38(2.5 \mathrm{I})$ \\
\hline$\geqslant$ US $\$ 40,000$ & $0.21(1.80)$ & $-1.53(2.57)$ & $2.93(2.83)$ \\
\hline Mean community SSS & $0.85(0.21)^{* * *}$ & $0.58(0.27)^{*}$ & $1.14(0.36)^{* *}$ \\
\hline \multicolumn{4}{|l|}{ Model 3} \\
\hline Age & $-0.02(0.04)$ & $-0.02(0.04)$ & $0.01(0.07)$ \\
\hline Not currently married & $1.60(1.02)$ & $1.15(1.29)$ & $1.23(1.78)$ \\
\hline Not currently employed & $-7.09(1.06)$ & $-7.27(1.3 \mathrm{I})$ & $-6.35(1.82)$ \\
\hline Mean highest education (years) & $0.39(0.20)$ & $0.47(0.26)$ & $0.43(0.34)$ \\
\hline \multicolumn{4}{|l|}{ Income ("missing"= referent) } \\
\hline Income $<$ US $\$ 40,000$ & $-1.61(1.39)$ & $-3.70(1.67)$ & $\mathrm{I} .4 \mathrm{I}(2.5 \mathrm{I})$ \\
\hline Income $\geqslant U S \$ 40,000$ & $-0.58(1.84)$ & $-2.71(2.64)$ & $2.48(2.85)$ \\
\hline Mean community SSS & $0.8 I(0.2 I)^{* * *}$ & $0.53(0.27)^{*}$ & $1.09(0.36)^{* *}$ \\
\hline \multicolumn{4}{|l|}{ MCS score } \\
\hline \multicolumn{4}{|l|}{ Model I } \\
\hline Age & $0.20(0.04)$ & $0.22(0.05)$ & $0.19(0.65)$ \\
\hline Not currently married & $-1.86(0.92)$ & $-1.01(1.21)$ & $-3.75(1.54)$ \\
\hline Not currently employed & $-2.97(0.98)$ & $-3.88(1.22)$ & $-2.05(1.66)$ \\
\hline Mean community SSS & $0.7 I(0.20) * *$ & $0.59(0.26)^{*}$ & $0.85(0.33)^{* *}$ \\
\hline \multicolumn{4}{|l|}{ Model 2a } \\
\hline Age & $0.20(0.04)$ & $0.22(0.05)$ & $0.20(0.07)$ \\
\hline Not currently married & $-1.91(0.93)$ & $-1.04(1.22)$ & $-3.80(1.56)$ \\
\hline Not currently employed & $-3.00(0.99)$ & $-3.92(1.24)$ & $-2.08(1.67)$ \\
\hline Mean highest education (years) & $-0.07(0.19)$ & $-0.09(0.25)$ & $-0.08(0.31)$ \\
\hline Mean community SSS & $0.72(0.20)^{* * *}$ & $0.60(0.26)^{*}$ & $0.87(0.34)^{* *}$ \\
\hline \multicolumn{4}{|l|}{ Model $2 b$} \\
\hline Age & $0.20(0.40)$ & $0.22(0.05)$ & $0.21(0.06)$ \\
\hline Not currently married & $-1.03(0.98)$ & $-0.77(1.26)$ & $-1.94(1.65)$ \\
\hline Not currently employed & $-2.34(1.02)$ & $-3.53(1.29)$ & $-1.02(1.69)$ \\
\hline
\end{tabular}


Table 3. (Continued)

\begin{tabular}{|c|c|c|c|}
\hline & Overall & Blacks & Whites \\
\hline \multicolumn{4}{|l|}{ Income ("missing" = referent) } \\
\hline$<$ US $\$ 40,000$ & $0.57(1.33)$ & $2.14(1.61)$ & $-2.04(2.33)$ \\
\hline$\geqslant$ US $\$ 40,000$ & $3.52(1.73)$ & $2.49(2.5 \mathrm{I})$ & $3.40(2.62)$ \\
\hline Mean community SSS & $0.68(0.20)^{* * *}$ & $0.62(0.26)^{*}$ & $0.67(0.33)^{*}$ \\
\hline \multicolumn{4}{|l|}{ Model 3} \\
\hline Age & $0.20(0.04)$ & $0.22(0.05)$ & $0.22(0.06)$ \\
\hline Not currently married & $-1.09(0.99)$ & $-0.77(1.27)$ & $-2.01(1.65)$ \\
\hline Not currently employed & $-2.36(1.02)$ & $-3.55(1.29)$ & $-1.07(1.70)$ \\
\hline Mean highest education (years) & $-0.17(0.19)$ & $-0.17(0.26)$ & $-0.24(0.31)$ \\
\hline \multicolumn{4}{|l|}{ Income ("missing"= referent) } \\
\hline Income $<$ US $\$ 40,000$ & $0.70(1.34)$ & $2.34(1.64)$ & $-2.05(2.33)$ \\
\hline Income $\geqslant U S \$ 40,000$ & $3.87(1.77)$ & $2.92(2.59)$ & $3.66(2.64)$ \\
\hline Mean community SSS & $0.70(0.20) * * *$ & $0.64(0.26) *$ & $0.70(0.34)^{*}$ \\
\hline
\end{tabular}

SSS: subjective social status.

aEffect estimates represent beta coefficients and their associated standard errors.

bParticipant race did not moderate the effects of OSS on mental health functioning or physical health functioning, nor did the inclusion of race by OSS interactions change the results for Community SSS.

***Within-group $p$ value $<0.00 \mathrm{I} ; *^{*}$ within-group $p$ value $<0.0 \mathrm{I}$; ${ }^{*}$ within-group $p$ value $<0.05$

and employment status in the overall sample. This positive association remained in the full model (Table 3, Model 3).

There was no evidence of effect modification by race on the association between community SSS and physical health functioning. The beta coefficients (standard errors) for Models 1-3 ranged from $0.60(0.42)$ to 0.56 $(0.42)$ with $p$ values for the interaction ranging between 0.15 and 0.18 . Likewise, there was no evidence of effect modification by race on the association between community SSS and mental health functioning. The beta coefficients (standard errors) for Models 1-3 ranged from $0.20(0.40)$ to $0.10(0.40)$ with $p$ values for the interaction ranging between 0.62 and 0.80 .

\section{SSS and physical and mental health functioning scores using US referent group}

Overall, we found a significant association between US SSS and PCS score when adjusting for age, marital status, and employment status (Table 4, Model 1), such that PCS score increased by 0.76 (SE $0.23 ; p<0.001$ ) for every 1 unit increase in SSS. This association was attenuated but remained significant in the fully adjusted model $(\beta=0.69$; SE 0.23; $p<0.003)$. The associations between US SSS and physical health functioning differed significantly by race, such that the PCS score was significantly greater for Whites compared to Blacks for each "rung" increase in US SSS ranking. The beta coefficients (standard errors) for Models 1-3 ranged from 1.24 (0.47) to $1.06(0.48)$ with $p$ values for the interaction ranging between $<0.01$ and 0.03 .

Similarly, as SSS in the United States increased, MCS scores increased by 0.81 (SE $0.22 ; p<0.001$; Model 1) in the overall sample. US SSS remained significantly associated with MCS score in the fully adjusted model (Model $3)$. The effect of having a higher perceived US SSS on mental health functioning was significantly greater in Whites than Blacks. The beta coefficients (standard errors) for Models 1-3 ranged from $1.32(0.45)$ to $1.16(0.45)$ with $p$ values for the interaction ranging between $<0.01$ and 0.01 . 
Table 4. Linear associations between US standing and physical component summary (PCS) and mental component summary (MCS) scores, overall and race-stratified.

\begin{tabular}{|c|c|c|c|}
\hline & Overall & Blacks & Whites \\
\hline \multicolumn{4}{|l|}{ PCS score } \\
\hline \multicolumn{4}{|l|}{ Model I } \\
\hline Age & $-0.02(0.04)$ & $-0.02(0.05)$ & $-0.02(0.07)$ \\
\hline Not currently married & $1.29(0.96)$ & $1.14(1.25)$ & $1.42(1.65)$ \\
\hline Not currently employed & $-7.44(1.02)$ & $-7.31(1.27)$ & $-6.90(1.75)$ \\
\hline Mean US SSS & $0.76(0.23)^{* * *}$ & $0.31(0.26)$ & $1.56(0.44)^{* * *}$ \\
\hline \multicolumn{4}{|l|}{ Model 2a } \\
\hline Age & $-0.01(0.04)$ & $-0.01(0.05)$ & $-0.01(0.07)$ \\
\hline Not currently married & $1.59(0.97)$ & $1.32(1.25)$ & $1.59(1.65)$ \\
\hline Not currently employed & $-7.23(1.02)$ & $-7.04(1.27)$ & $-6.76(1.75)$ \\
\hline Mean highest education (years) & $0.44(0.20)$ & $0.48(0.25)$ & $0.37(0.34)$ \\
\hline Mean US SSS & $0.72(0.22)^{* *}$ & $0.31(0.26)$ & $1.45(0.45)^{* *}$ \\
\hline \multicolumn{4}{|l|}{ Model 2b } \\
\hline Age & $-0.02(0.04)$ & $-0.03(0.05)$ & $-0.01(0.07)$ \\
\hline Not currently married & $1.62(1.03)$ & $1.14(1.30)$ & $1.53(1.77)$ \\
\hline Not currently employed & $-7.36(1.06)$ & $-7.49(1.32)$ & $-6.55(I .8 I)$ \\
\hline \multicolumn{4}{|l|}{ Income ("missing"= referent) } \\
\hline$<$ US $\$ 40,000$ & $-1.43(1.40)$ & $-3.39(1.67)$ & $\mathrm{I} .84(2.5 \mathrm{I})$ \\
\hline$\geqslant$ US $\$ 40,000$ & $-0.01(1.82)$ & $-1.76(2.59)$ & $2.50(2.84)$ \\
\hline Mean US SSS & $0.72(0.23) \dagger$ & $0.27(0.27)$ & $1.52(0.46)^{* *}$ \\
\hline \multicolumn{4}{|l|}{ Model 3} \\
\hline Age & $-0.01(0.04)$ & $-0.01(0.05)$ & $-0.01(0.07)$ \\
\hline Not currently married & $1.76(1.03)$ & $1.17(1.30)$ & $1.60(1.77)$ \\
\hline Not currently employed & $-7.28(1.06)$ & $-7.36(1.31)$ & $-6.49(1.81)$ \\
\hline Mean highest education (years) & $0.45(0.20)$ & $0.57(0.26)$ & $0.35(0.34)$ \\
\hline \multicolumn{4}{|l|}{ Income ("missing"= referent) } \\
\hline Income $<$ US $\$ 40,000$ & $-1.74(1.40)$ & $-4.00(1.68)$ & $\mathrm{I} .83(2.5 \mathrm{I})$ \\
\hline Income $\geqslant U S \$ 40,000$ & $-0.90(1.86)$ & $-3.16(2.66)$ & $2.17(2.85)$ \\
\hline Mean US SSS & $0.69(0.23)^{* *}$ & $0.27(0.26)$ & $1.44(0.47)^{* *}$ \\
\hline \multicolumn{4}{|l|}{ MCS score } \\
\hline \multicolumn{4}{|l|}{ Model I } \\
\hline Age & $0.20(0.04)$ & $0.23(0.05)$ & $0.14(0.07)$ \\
\hline Not currently married & $-1.81(0.92)$ & $-1.19(1.21)$ & $1.51(-2.84)$ \\
\hline Not currently employed & $-3.08(0.98)$ & $-4.17(1.23)$ & $-1.37(1.60)$ \\
\hline Mean US SSS & $0.81(0.22)^{* * *}$ & $0.36(0.26)$ & $1.80(0.40)^{* * *}$ \\
\hline \multicolumn{4}{|l|}{ Model 2a } \\
\hline Age & $0.20(0.04)$ & $0.23(0.05)$ & $0.13(0.07)$ \\
\hline Not currently married & $-1.85(0.93)$ & $-1.21(1.22)$ & $-2.97(1.52)$ \\
\hline Not currently employed & $-3.10(0.98)$ & $-4.19(1.24)$ & $-1.48(1.61)$ \\
\hline Mean highest education (years) & $-0.06(0.19)$ & $-0.04(0.25)$ & $-0.28(0.31)$ \\
\hline Mean US SSS & $0.81(0.22)^{* * *}$ & $0.36(0.26)$ & $I .89(0.4 I)^{* *}$ \\
\hline \multicolumn{4}{|l|}{ Model 2b } \\
\hline Age & $0.20(0.04)$ & $0.23(0.05)$ & $0.16(0.07)$ \\
\hline Not currently married & $-1.06(0.98)$ & $-0.98(1.27)$ & $-1.64(1.61)$ \\
\hline Not currently employed & $-2.5 \mathrm{I}(\mathrm{I} .0 \mathrm{I})$ & $-3.86(1.29)$ & $-0.69(1.65)$ \\
\hline
\end{tabular}


Table 4. (Continued)

\begin{tabular}{|c|c|c|c|}
\hline & Overall & Blacks & Whites \\
\hline \multicolumn{4}{|l|}{ Income ("missing"= referent) } \\
\hline$<$ US $\$ 40,000$ & $0.64(1.33)$ & $2.09(1.62)$ & $-1.48(2.28)$ \\
\hline$\geqslant$ US $\$ 40,000$ & $3.33(1.73)$ & $2.34(2.53)$ & $2.55(2.58)$ \\
\hline Mean US SSS & $0.75(0.22)^{* * *}$ & $0.37(0.26)$ & $1.54(0.42)^{* * *}$ \\
\hline \multicolumn{4}{|l|}{ Model 3} \\
\hline Age & $0.20(0.04)$ & $0.23(0.05)$ & $0.16(0.07)$ \\
\hline Not currently married & $-1.11(0.98)$ & $-0.98(1.27)$ & $-1.72(1.61)$ \\
\hline Not currently employed & $-2.53(1.01)$ & $-3.89(1.29)$ & $-0.77(1.65)$ \\
\hline Mean highest education (years) & $-0.15(0.19)$ & $-0.11(0.26)$ & $-0.38(0.31)$ \\
\hline \multicolumn{4}{|l|}{ Income ("missing" = referent) } \\
\hline Income $<$ US $\$ 40,000$ & $0.75(\mathrm{I} .34)$ & $2.21(1.65)$ & $-1.47(2.28)$ \\
\hline Income $\geqslant U S \$ 40,000$ & $3.63(1.78)$ & $2.6 I(2.6 I)$ & $2.90(2.59)$ \\
\hline Mean US SSS & $0.76(0.22) * * *$ & $0.37(0.26)$ & $1.63(0.42)^{* * *}$ \\
\hline
\end{tabular}

SSS: subjective social status.

aEffect estimates represent beta coefficients and their associated standard errors.

bParticipant race did not moderate the effects of OSS on mental health functioning or physical health functioning, nor did the inclusion of race by OSS interactions change the results for US social standing.

***Within-group $p$ value $<0.00 \mathrm{I}$; ***within-group $p$ value $<0.0 \mathrm{I}$; *within-group $p$ value $<0.05$

\section{Discussion}

Our study generated four main findings. First, SSS measured using both the community and US ladders was associated with better physical and mental health functioning in the overall sample independent of educational level, household income or both. Second, the effect of SSS on physical and mental health functioning differed significantly by race, but only when using the US referent group. When the associations differed, they were stronger for Whites than Blacks. Third, there were significant racial differences in mean SSS and OSS measures, with Blacks having higher SSS ratings on both the US and community ladders, despite having less education and more individuals with annual household income $<$ US\$40,000. Finally, correlations between SSS and OSS are generally poor, especially among Blacks, and racial differences in correlations are only significant for the US, not community SSS.

\section{SSS and physical and mental health functioning}

Like many, but not all studies (Adler et al., 2008; Singh-Manoux et al., 2005), we found that SSS remained independently associated with PCS and MCS scores after adjustment for OSS indicators (education and income). Only one other published study used the same outcome measure (SF-12) to assess physical and mental health functioning (Adler et al., 2008). This study, conducted in individuals of Mexican origin living in Texas, also demonstrated a positive linear association between SSS, PCS, and MCS scores. Our findings are also consistent with other studies which used a single-item overall self-rated health measure (Adler et al., 2000, 2008; Demakakos et al., 2008; Singh-Manoux et al., 2003, 2005; Thompson et al., 2014; Wright and Steptoe, 2005) and studies which used other measures (primarily measures of depressive symptoms) to assess mental health functioning (Adler et al., 2000; Ostrove et al., 1999; SinghManoux et al., 2003; Subramanyam et al., 2012).

In race-stratified analyses, higher SSS was independently associated with higher PCS and MCS scores in both Blacks and Whites. However, the significance of the differences varied by race and referent group. Among Blacks, SSS was significantly associated with both PCS and MCS scores using the community as the referent group (within-group $p$ values $<0.05$ ); however, the 
associations did not differ using the United States as the referent (within-group $p$ values $>0.05$ ). Among Whites, the associations between SSS with PCS and MCS scores were statistically significant using both the community and US referent groups (within-group $p$ values $<0.05$ ).

Although we did not have a priori hypotheses related to effect modification by race on the association between SSS and physical and mental health functioning, we found that race was an effect modifier and the strength of the association was sensitive to the reference group used. Associations between community SSS with PCS and MCS scores were not statistically different by race. In other words, the effect of perceiving equitable or better social standing compared to others in one's community on selfrated physical and mental health functioning is the same for Blacks and Whites. However, the association between US SSS with PCS and MCS scores was stronger in Whites than Blacks. Taken together, these findings suggest that both perceptions of value in one's community and advantage in terms of money, education, and job type compared to others in society are important contributors to how individuals assess their health status. However, for Whites, how they compare to others in society with respect to more objective measures of social status is more influential on their self-ratings of health-related quality of life than it is for Blacks. Subramanyam et al. (2012) also assessed SSS using a proximal ("community") and distal ("US") referent group in their study; however, they only sampled Blacks and therefore could not examine effect modification by race.

\section{Subjective versus OSS}

Similar to other researchers (Kaufman et al., 1998; Subramanyam et al., 2012) we found that mean SSS scores were higher using the community versus the US ladder. Moreover, Blacks rated their community SSS higher than Whites, despite having a lower mean educational level and a higher percentage of participants with annual income $<$ US $\$ 40,000$. Wolff et al. (2010), using a nationally representative US sample, also assessed multiple referent groups in examining whether race and referent group influenced SSS ratings. Unlike our findings, they found that mean scores for US SSS were significantly higher for Whites than Blacks and that there were no racial differences in mean SSS scores using a proximal referent ("your neighbors"). On the contrary, we found that Blacks had higher SSS ratings than Whites using both a proximal and distal referent. Methodological differences in our samples and unmeasured local contextual factors may explain why our findings differed from theirs. Social comparison research suggests that using referents with greater similarity or proximity to the respondent may be more salient to racial and ethnic minorities when they compare themselves to others (Gibbons and Gerrard, 1991; Stiles and Kaplan, 2004).

We found a weaker correlation between SSS and OSS measures using the community ladder, as compared to the US ladder. How individuals define "community" is unclear and may differ by race, particularly given the high levels of residential and neighborhood segregation that exist in the Southeastern United States among racial/ethnic minorities. Segregation by race/ ethnicity tends to be much stronger than segregation by income (Acevedo-Garcia et al., 2003). Moreover, correlations were stronger among Whites than Blacks. The weaker correlation between SSS and OSS measures in Blacks is consistent with prior research (Kaufman et al., 1998; Ostrove et al., 1999; Singh-Manoux et al., 2005; Subramanyam et al., 2012). These findings suggest that SSS ratings are not driven by OSS indicators in Blacks. Social status may be a more complex and nuanced issue for Blacks, particularly those in the rural South.

Like all research, our study has limitations. First, the data are cross-sectional; therefore, we cannot assess the directionality of the associations or make causal inferences. However, we are collecting longitudinal data on SSS, physical, and mental health functioning and can assess directionality in future studies. Second, since these data are not from a nationally representative sample, generalizability may be limited. Third, we did not objectively measure physical 
functioning; health assessments were based on respondent self-report and may be subject to measurement bias. Fourth, the alpha coefficients for the PCS and MCS subscales are lower than has been reported in other studies. Fifth, there may be unmeasured confounders, such as psychological well-being, general sense of selfworth, or depression that influences ratings of SSS as well as physical and mental health functioning. However, other studies have shown that the correlation between SSS and health persists even after controlling for these factors. SSS may provide a more patient-centered metric for assessing the effects of social resources on health behaviors and outcomes; however, the implications of assessing SSS at the "point of care" and how SSS would be used in decision-making in clinical settings remains unclear. This is an area that is ripe for additional research.

Despite these limitations, our study adds to the limited research on SSS and its effect on health overall and in racial/ethnic minorities specifically. Blacks accounted for 60 percent of our study sample - a greater percentage than many other published studies examining similar questions. Our study is one of the few toassesses SSS using both a proximal and distal referent group; thereby allowing us to more fully understand the relationships between the two and their differential effects on health (Goodman et al., 2007; Kaufman et al., 1998; MacArthur Research Network on Socioeconomic Status and Health, 2008; Subramanyam et al., 2012; Wright and Steptoe, 2005). SSS measures are quick and easy to administer, making them feasible for use in conducting research. This study has important implications for health equity research. SSS, assessed using a proximal referent in particular, may reflect a source of social capital which is health promoting. Social capital is important in racial/ethnic minority groups who otherwise appear to be "disadvantaged" when only OSS indicators are considered. As such, SSS measures may be particularly useful in furthering health equity research to understand socio-psychological factors that affect health. More research is needed to understand what factors drive the SSS ratings in Blacks and how those ratings vary depending on social, cultural, and contextual factors.

\section{Acknowledgements}

We would like to acknowledge the entire Heart Healthy Lenoir Research team for their invaluable contributions to this project. We also want to thank the providers and staff at the practices that took part in this study.

\section{Declaration of conflicting interests}

The author(s) declared no potential conflicts of interest with respect to the research, authorship, and/or publication of this article.

\section{Funding}

The author(s) disclosed receipt of the following financial support for the research, authorship, and/or publication of this article: This study was funded by the National Heart, Lung, and Blood Institute of the National Institutes of Health via award number NHLBI (1P50HL10584-01). The funding body had no role in the collection, analysis, and interpretation of study data, nor did they play a role in the writing of the manuscript or the submission of the manuscript for publication. Dr Crystal Cené's work on this project was supported by the National Center for Research Resources (grant number KL2RR025746) and the National Heart, Lung, and Blood Institute (grant number K23HL107614).

\section{References}

Acevedo-Garcia D, Lochner KA, Osypuk TL, et al. (2003) Future directions in residential segregation and health research: A multilevel approach. American Journal of Public Health 93(2): 215-221.

Adler NE, Epel ES, Castellazzo G, et al. (2000) Relationship of subjective and objective social status with psychological and physiological functioning: Preliminary data in healthy White women. Health Psychology: Official Journal of the Division of Health Psychology, American Psychological Association 19(6): 586-592.

Adler NE, Singh-Manoux A, Schwartz J, et al. (2008) Social status and health: A comparison of British civil servants in Whitehall-II with European- and African-Americans in CARDIA. Social Science \& Medicine 66(5): 1034-1045.

Anderson C, Kraus MW, Galinsky AD, et al. (2012)

The local-ladder effect: Social status and 
subjective well-being. Psychological Science 23(7): 764-771.

Cohen S, Alper CM, Doyle WJ, et al. (2008) Objective and subjective socioeconomic status and susceptibility to the common cold. Health Psychology 27(2): 268-274.

Cornman JC, Goldman N, Glei DA, et al. (2003) Social ties and perceived support: Two dimensions of social relationships and health among the elderly in Taiwan. Journal of Aging and Health 15(4): 616-644.

Demakakos P, Nazroo J, Breeze E, et al. (2008) Socioeconomic status and health: The role of subjective social status. Social Science \& Medicine 67(2): 330-340.

Finkelstein DM, Kubzansky LD and Goodman E (2006) Social status, stress, and adolescent smoking. Journal of Adolescent Health 39(5): 678-685.

Franzini L and Fernandez-Esquer ME (2006) The association of subjective social status and health in low-income Mexican-origin individuals in Texas. Social Science \& Medicine 63(3): 788-804.

Gibbons FX and Gerrard M (1991) Downward social comparison and coping with threat. In: Suls JM and Wills TA (eds) Social Comparison: Theory and Research. Hillsdale, NJ: Lawrence Erlbaum, pp. 317-345.

Goodman E, Huang B, Schafer-Kalkhoff T, et al. (2007) Perceived socioeconomic status: A new type of identity that influences adolescents' self-rated health. Journal of Adolescent Health 41(5): 479-487.

Halladay JR, Donahue KE, Hinderliter A, et al. (2013) The heart healthy Lenoir project-an intervention to reduce disparities in hypertension control: Study protocol. BMC Health Services Research 13: 441-452.

$\mathrm{Hu}$ P, Adler NE, Goldman N, et al. (2005) Relationship between subjective social status and measures of health in older Taiwanese persons. Journal of the American Geriatrics Society 53(3): 483-488.

Kaufman JS, Long AE, Liao Y, et al. (1998) The relation between income and mortality in US blacks and whites. Epidemiology 9: 147-155.

Kraus MW and Stephens NM (2012) A road map for an emerging psychology of social class. Social \& Personality Psychology Compass 6(9): 642-656.

MacArthur Research Network on Socioeconomic Status and Health (2008) Sociodemographic Questionnaire. Available at: www.macses.ucsf. edu/research/psychosocial/subjective
Operario D, Adler NE and Williams DR (2004) Subjective social status: Reliability and predictive utility for global health. Psychology \& Health 19(2): 237-246.

Ostrove JM, Adler NE, Kuppermann M, et al. (2000) Objective and subjective assessments of socioeconomic status and their relationship to self-rated health in an ethnically diverse sample of pregnant women. Health Psychology: Official Journal of the Division of Health Psychology, American Psychological Association 19(6): 613-618.

Ostrove JM, Feldman P and Adler NE (1999) Relations among socioeconomic status indicators and health for African-Americans and Whites. Journal of Health Psychology 4(4): 451-463.

Singh-Manoux A, Adler NE and Marmot MG (2003) Subjective social status: Its determinants and its association with measures of ill-health in the Whitehall II study. Social Science \& Medicine 56(6): 1321-1333.

Singh-Manoux A, Marmot MG and Adler NE (2005) Does subjective social status predict health and change in health status better than objective status? Psychosomatic Medicine 67(6): 855-861.

Stiles BL and Kaplan HB (2004) Adverse social comparison processes and negative self-feelings: A test of alternative models. Social Behavior and Personality: An International Journal 32(1): 31-44.

Subramanyam MA, Diez-Roux AV, Hickson DA, et al. (2012) Subjective social status and psychosocial and metabolic risk factors for cardiovascular disease among African Americans in the Jackson Heart Study. Social Science \& Medicine 74(8): 1146-1154.

Thompson MG, Gaglani MJ, Naleway A, et al. (2014) Changes in self-rated health and subjective social status over time in a cohort of healthcare personnel. Journal of Health Psychology 19(9): 1185-1196.

Ware J Jr, Kosinski M and Keller SD (1996) A 12-Item Short-Form Health Survey: Construction of scales and preliminary tests of reliability and validity. Medical Care 34(3): 220-233.

Wolff LS, Acevedo-Garcia D, Subramanian SV, et al. (2010) Subjective social status, a new measure in health disparities research: Do race/ ethnicity and choice of referent group matter? Journal of Health Psychology 15(4): 560-574.

Wright CE and Steptoe A (2005) Subjective socioeconomic position, gender and cortisol responses to waking in an elderly population. Psychoneuroendocrinology 30(6): 582-590. 